\title{
Los equinodermos (Echinodermata) del arrecife de Cabo Pulmo, Pacífico de México
}

\author{
Carlos E. Cintra Buenrostro ${ }^{1}$, Héctor Reyes Bonilla 1, Oscar Arizpe Covarrubias 1
}

Universidad Autónoma de Baja California Sur. Departamento de Biología Marina. Apartado postal 19-B, CP 23080. La Paz, B.C.S., México.

\author{
Recibido 6-II-1997. Corregido 1-XII-1997. Aceptado 14-I-1998.
}

\begin{abstract}
A systematic list of the echinoderms from Cabo Pulmo reef, Pacific coast of México $\left(23.5^{\circ} \mathrm{N}\right)$, is presented. Data were obtained from field surveys (1986 to 1993), and complemented with literature searches. A total of 38 species have been reported at Cabo Pulmo, but only 25 of them were actually observed in the reef during our visits. This finding suggests that community structure and composition have changed in important ways since 1943, date of the first reports of echinoderms for the locality.
\end{abstract}

Key words: Cabo Pulmo, Gulf of California, México, Echinodermata, Asteroidea, Ophiuroidea, Echinoidea, Holothuroidea.

El estudio de la fauna de equinodermos del Pacífico de México se ha enfocado a la realización de inventarios faunísticos (Solís Marín et al. 1993). Siguiendo esta tradición, se presenta el listado de las especies de equinodermos registradas dentro del sistema arrecifal de Cabo Pulmo, con base en datos obtenidos en el campo entre 1986 y 1993, y una revisión extensa de la literatura. El arrecife de Cabo Pulmo fue decretado como Parque Marino Nacional en 1995 debido a su importancia como ecosistema dentro del Golfo de California y ya ha sido bien descrito (Arizpe Covarrubias 1997). Para este trabajo se realizó una búsqueda intensiva de equinodermos, la cual se hizo de manera no destructiva, durante el día y exclusivamente dentro de la zona arrecifal, usando buceo libre y autónomo. La identificación de las especies estuvo basada principalmente en Deichmann (1941, 1958), Caso (1978, $1979,1992)$ y Brusca (1980).

En el arrecife se han registrado 38 especies del Phylum Echinodermata (Cuadro 1), aunque durante nuestras visitas solo se observaron 25, siendo el erizo Hesperocidaris asteriscus Clark, 1948 un nuevo registro para la localidad. Es posible que muchas especies crípticas (por ejemplo, los erizos irregulares y los ofiuroideos que viven dentro de las cabezas de coral) estén subrepresentadas al no haber sido estudiados sus hábitats principales, y por el tipo de muestreo realizado. Por ello, el listado real de especies de equinodermos de Cabo Pulmo debe estar aún incompleto.

La revisión de la literatura evidenció un cambio importante en la composición y estruc- 


\section{CUADRO 1.}

Listado sistemático de los equinodermos del arrecife de Cabo Pulmo

\section{Phylum Echinodermata}

Clase Asteroidea

Orden Valvatida

Familia Asterodiscididae Amphiaster insignis Verrill, 1868 (2)

Familia Oreasteridae Nidorellia armata (Gray, 1840) $(1,6)$

Pentaceraster cumingi (Gray, 1840) $(1,6)$

Familia Ophidiasteridae

Linckia columbiae Gray, $1840(4,6)$

Pharia pyramidata (Gray, 1840) $(1,2,5,6)$

Phataria unifascialis (Gray, 1840) $(1,2,4,5,6)$

Familia Mithrodiidae Mithrodia bradleyi Verrill, $1867(1,5,6)$

Familia Acanthasteridae Acanthaster planci (Linnaeus, 1758) $(2,5,6)$

Orden Spinulosida

Familia Echinasteridae Echinaster (Othilia) tenuispina Verrill, 1871 (2) Henricia sp. (2)

Orden Forcipulatida

Familia Heliasteridae Heliaster kubiniji Xantus, $1860(2,6)$

Familia Asteriidae Astrometis sertulifera (Xantus, 1860) (4)

\section{Clase Ophiuroidea}

Orden Ophiurida

Familia Ophiactidae Ophiactis simplex (Le Conte, 1851) $(1,2,6)$

Familia Ophiotrichidae Ophiothrix rudis Lyman, 1874 (1) Ophiothrix spiculata Le Conte, 1851 (1)

Familia Ophiocomidae Ophiocoma aethiops Lutken, $1859(2,6)$ Ophiocoma alexandri Lyman, $1860(1,2,6)$

Familia Ophionereidae Ophionereis annulata (Le Conte, 1851) $(2,6)$

Familia Ophiodermatidae

Diopederma danianum (Verrill, 1867) $(2,3,6)$ Ophioderma teres (Lyman,1860) (6)

\section{Clase Echinoidea}

Orden Cidaroida

Familia Cidaridae

Eucidaris thouarsii (Valenciennes, 1846) $(1,2,5,6)$ Hesperocidaris asteriscus H.L. Clark, 1948 (6)

Orden Diadematoida

Familia Diadematidae

Centrostephanus coronatus (Verrill, 1867) $(5,6)$

Diadema mexicanum A. Agassiz, 1863 (1, 2, 5, 6)

Orden Arbacioida

Familia Arbaciidae Arbacia incisa (A. Agassiz, 1863) $(1,5,6)$
CUADRO 1. CONTINUACION

Listado sistemático de los equinodermos del arrecife de Cabo Pulmo

Orden Temnopleuroida

Familia Toxopneustidae Toxopneustes roseus (A. Agassiz, 1863) $(2,3,5,6)$ Tripneustes depressus A. Agassiz, $1863(2,3,6)$

Orden Echinoida

Familia Echinometridae

Echinometra vanbrunti A. Agassiz, $1863(1,2,5,6)$

Clase Holothuroidea

Orden Dendrochirotida

Familia Sclerodactylidae

Afrocucumis ovulum (Selenka, 1867) (1)

Neothyone gibbosa Deichmann, $1941(2,3,6)$

Orden Aspidochirotida

Familia Holothuriidae

Holothuria (Halodeima) kefersteini (Selenka, 1867) (2)

Holothuria (Selenkothuria) lubrica Selenka, $1867(2,6)$

Holothuria (Thymiosycia) impatiens (Forskaal,1775)(2)

Holothuria (Vaneyothuria) zacae Deichmann, 1937 (2)

Labidodemas americanum ? Deichmann, 1938 (2)

Familia Stichopodidae

Isostichopus fuscus (Ludwig, 1875) $(2,5,6)$

Orden Apodida

Familia Synaptidae

Euapta godeffroyi (Semper, 1868) $(2,4,6)$

Familia Chiridotidae

Chiridota aponocrita H.L. Clark, 1920 (2)

Referencias sobre registros de las especies: 1) Steinbeck \& Ricketts 1941; 2) Brusca \& Thomson 1975; 3) Brusca 1980; 4) Encarnación \& Keer García 1991; 5) Baynes 1993; 6) Datos de campo.

tura de la comunidad, al existir al menos 13 táxones previamente registrados, cuya presencia no ha sido confirmada en los últimos 20 o 50 años (Steinbeck \& Ricketts 1941, Brusca \& Thomson 1975). Las especies faltantes no son explotables, por lo que su desaparición debe tener causas naturales o bien, puede ser parte de un efecto de cadena, ya que está documentada la extinción local o disminución poblacional de varias especies de invertebrados por sobreexplotación pesquera (Arizpe Covarrubias en prep.); tales daños pueden haber alterado la red trófica local y afectado a los equinodemos. Sin embargo, no se puede descartar que la desaparición nominal de algunas especies sea también un efecto del muestreo, debido a su 
selectividad. Esta incertidumbre obliga a la continuación de los estudios y remarca la importancia de investigaciones que se lleven a cabo en intervalos grandes de tiempo, como formas más adecuadas para conocer el estado de las comunidades.

El estudio se llevó a cabo gracias al apoyo de la DGICSA-SEP (1987) y la UABCS (1989-1993), así como con la colaboración de la familia Castro Fiol, residentes locales, y de un gran número de técnicos y estudiantes del Departamento de Biología Marina de la UABCS. Se agradecen también las sugerencias de dos árbitros anónimos, que mejoraron la presentación y contenido del trabajo.

\section{RESUMEN}

Este trabajo presenta una lista sistemática de los equinodermos del arrecife de Cabo Pulmo, México. Los datos fueron obtenidos de revisiones de literatura y labores de campo llevadas a cabo entre los años de 1986 a 1993. Un total de 38 especies han sido registradas para Cabo Pulmo, pero sólo 25 de ellas fueron observadas directamente en el campo durante nuestras visitas, lo que sugiere que la estructura y composición de la comunidad han cambiado de forma importante desde 1943, fecha de los primeros registros de equinodermos para la localidad.

\section{REFERENCIAS}

Arizpe Covarrubias, O. 1997. The northernmost coral reef in the eastern Pacific. Proc. 8th Int. Coral Reef Symp., Panamá. En prensa.

Baynes, T.W. 1993. Effects of sedimentation, light, and grazing on the encrusting community of a tropical rock reef in the southern Gulf of California. Tesis Doctoral, University of California, San Diego, California.
Brusca, R.C. 1980. Common intertidal invertebrates of the Gulf of California. University of Arizona, Tucson, Arizona. $513 \mathrm{p}$.

Brusca, R.C. \& D.A. Thomson. 1975. Pulmo reef: the only "coral reef" in the Gulf of California. Ciencias Marinas 1: 37-53.

Caso, M.E. 1978. Los equinoideos del Pacífico de México. Parte 1. Ordenes Cidaroidea y Aulodonta. Parte 2. Ordenes Stiridonta y Camarodonta. Publ. Espec. An. Centro Cienc. Mar Limnol., UNAM 1: 1-244.

Caso, M.E. 1979. Los equinodermos de la Bahía de Mazatlán, Sinaloa. An. Centro Cienc. Mar y Limnol., UNAM. 6: 197-368.

Caso, M.E. 1992. Los equinodermos asteroideos, ofiuroideos y equinoideos de la Bahía de Mazatlán, Sinaloa. An. Inst. Cienc. Mar y Limnol., UNAM, Publ. Esp. 11: 1-214.

Deichmann, E. 1941. The Holothuroidea collected by the "Velero" III during the years 1932 to 1938. Part I, Dendrochirotida. Allan Hancock Pac. Exped. 8: 61-195.

Deichmann, E. 1958. The Holothuroidea collected by the "Velero" III and IV during the years 1932 to 1954. Part II, Aspidochirota. Allan Hancock Pac. Exped. 11: 253-349.

Encarnación, R.D. \& S. Keer García. 1992. Compuestos con actividad antimicrobiana de organismos marinos. Rev. Mex. Cienc. Farmac. 22: 33-41.

Solís Marín, F.A., M.D. Herrero Pérezrul, A. Laguarda Figueras \& J. Torres Vega. 1993. Asteroideos y equinoideos (Echinodermata) de México. p. 91-105. In S.I. Salazar Vallejo \& N.E. González (eds.). Biodiversidad marina y costera de México. CONABIO/ CIQRO. Chetumal, México.

Steinbeck, J. \& E.F. Ricketts. 1941. Sea of Cortez. Viking, Nueva York. 598 p. 